\title{
Delayed Lactogenesis II is Associated With Lower Sleep Efficiency and Greater Variation in Nightly Sleep Duration in the Third Trimester
}

Journal of Human Lactation $1-12$

(C) The Auchor(s) 2019

Article reuse guidelines: sagepub.com/journals-permissions DOI: 10.117710890334419830991 journals.sagepub.com/home/jhl (S)SAGE

\author{
Theresa Casey, PhD ${ }^{1}$ 稚, Hui Sun, BS $^{2}$, Helen J. Burgess, PhD $^{3}$, \\ Jennifer Crodian, BS', Shelley Dowden, BS ${ }^{4}$, Shelby Cummings, BS', \\ Karen Plaut, PhD', David Haas, MD', Lingsong Zhang, PhD ${ }^{2,5}$, \\ and Azza Ahmed, DNSc ${ }^{6}$
}

\begin{abstract}
Background. Metabolic and hormonal disturbances are associated with sleep disturbances and delayed onset of lactogenesis II. Research Aims. The aim of this study was to measure sleep using wrist actigraphy during gestation weeks 22 and 32 to determine if sleep characteristics were associated with blood glucose, body mass index, gestational related disease, delayed onset of lactogenesis II, or work schedule.

Methods. Demographic data were collected at study intake from primiparous women who wore a wrist actigraph during gestation weeks $22(n=50)$ and $32(n=44)$. Start and end sleep time, total nighttime sleep, sleep efficiency, wake after sleep onset, and sleep fragmentation were measured. Night to night variability was assessed with the root mean square of successive difference. Blood glucose levels, body mass index, and gestational disease data were abstracted from medical charts. Timing of lactogenesis II was determined by survey.

Results. Between gestation week 22 and 32, sleep efficiency decreased and fragmentation increased $(p<.05)$. During gestation week 32, blood glucose was negatively correlated with sleep duration, and positively related to fragmentation $(p<.05)$. Women who experienced delayed lactogenesis II had lower sleep efficiency and greater fragmentation $(p<.05)$, and greater night-to-night variability in sleep start and end time, efficiency, and duration during gestation week $32(p<.05)$. Conclusion. Women with better sleep efficiency and more stable nightly sleep time are less likely to experience delayed onset of lactogenesis II. Interventions to improve sleep may improve maternal health and breastfeeding adequacy.
\end{abstract}

\section{Keywords}

Breastfeeding, lactation, lactogenesis, maternal physiology

\section{Background}

Delayed lactogenesis II (DLII) is milk "coming in" more than 72 hours after birth (Lawrence \& Lawrence, 2016; Brownell, Howard, Lawrence \& Dozier, 2012 ). Infants of women who experience DLII are 7-times more likely to lose excessive weight the first five days postpartum (Dewey, Nommsen-Rivers, Heinig \& Cohen, 2003; Nommsen-Rivers, 2016), and thus it is not surprising that DLII is associated with increased risk for early formula supplementation and premature breastfeeding cessation (Brownell et al., 2012).

There appears to be a metabolic-hormonal link to DLII, as risks for DLII include cesarean delivery, long labor, elevated blood cortisol, overweight/obesity, and high infant birth weight (Fu et al., 2015; Preusting, Brumley,

\footnotetext{
'Department of Animal Sciences, Purdue University, West Lafayette, IN, USA

${ }^{2}$ Department of Statistics, Purdue University, West Lafayette, IN, USA

${ }^{3}$ Department of Behavioral Sciences, Rush University Medical Center, Chicago, IL, USA

'Department of Obstetrics and Gynecology, Indiana University, Indianapolis, IN, USA

${ }^{5}$ Regenstrief Center for Healthcare Engineering. Purdue University, West Lafayette, IN, USA

${ }^{6}$ School of Nursing, Purdue University, West Lafayette, IN, USA

Date submitted: March 5, 2018; Date accepted: January 23, 2019.

Corresponding Author:

Theresa M. Casey, PhD, Department of Animal Sciences, Purdue University, 615 W. State Street, West Lafayette, IN 47907-2050, USA.

Email: theresa-casey@purdue.edu
} 
Odibo, Spatz \& Louis, 2017). Lower glucose tolerance also has been associated with longer time to onset of lactogenesis II (Nommsen-Rivers, Dolan \& Huang, 2012), with diagnosis of gestational diabetes mellitus (GDM) being associated with DLII (Morceli et al., 2011). Moreover, prolactin release in response to suckling was significantly lower in overweight or obese women compared to healthy weight women in the early postpartum period, which may lead to failed lactogenesis II (Rasmussen \& Kjolhede, 2004; Ramiandrasoa et al., 2013). Older age at first pregnancy and primiparity are risks for DLII, with the rate among primiparas reported to range from $17.44 \%$ (Dewey et al., 2003; Matias, Nommsen-Rivers, Creed-Kanashiro \& Dewey, 2010; Nommsen-Rivers, Chantry, Peerson, Cohen \& Dewey, 2010).

Sleep disturbance during pregnancy is associated with metabolic and hormonal disturbances in women. Pregnant women who self-reported shorter nighttime sleep had an increased risk for impaired glucose tolerance (Facco, Grobman, Kramer, Ho \& Zee, 2010; Herring et al., 2014). An inverse association was found between self-reported nighttime sleep duration and blood glucose levels in midpregnancy (Reutrakul et al., 2011). Self-reported sleep disturbance in early pregnancy was associated with higher risk for the development of hyperglycemia (Izci, Jackson, Ratcliffe, Pack \& Pien, 2013). Self-reported poor sleep quality and short nocturnal sleep duration were independently associated with increased risk of GDM (Cai et al., 2017 ), and was increased among women reporting very short sleep $(<4 \mathrm{hr})$ compared with women sleeping at least 9 hours per night during early pregnancy (Qiu, Enquobahrie, Frederick, Abetew \& Williams, 2010). Pregnant women sleeping more than 8-9 hours or less than 8-9 hours had increased risk for GDM (Rawal, Hinkle, Zhu, Albert \& Zhang, 2017). Similarly, compared to 7-9 hours of sleep each day, the odds ratios for GDM in women who selfreported sleep duration of 9 hours or more per day or less than $<7$ hours/day was 1.21 or 1.36 , respectively (Wang et al., 2017). Moreover, self-reported sleep duration in early gestation ( $\sim 14$ weeks) was related to third trimester blood pressure and the development of hypertensive disorders (Williams et al., 2010).

Objectively measured sleep duration was associated with the development of GDM in nulliparous women (Facco et al., 2017). In a prospective study of women with GDM, sleep duration was negatively associated with fasting and one-hour postprandial blood glucose concentrations (Twedt, Bradley, Deiseroth, Althouse \& Facco, 2015). Moreover, short duration at approximately 21 weeks gestation was predictive of higher blood glucose values (Herring et al., 2014).

The National Institutes of Health (NIH) recommend that adults sleep 7-8 hours per day. Approximately $30 \%$ of adults in the US sleep six hours or less a day (Schoenborn, Adams \& Peregoy 2013). In the US, race and socio-economic level

\section{Key Messages}

- Sleep disturbance alters metabolism and hormonal milieu, and is associated with the development of gestational related diseases.

- Metabolic and hormonal disturbances during pregnancy, including obesity, hyperglycemia, gestational diabetes mellitus, and elevated cortisol, are associated with delayed onset of lactogenesis II.

- Using wrist actigraphy to measure sleep, we found that women who experienced delayed lactogenesis II had lower sleep efficiency and more variation in nightly sleep duration, as well as higher sleep fragmentation in the third trimester.

- This is a novel finding, and suggests that interventions that target sleep management during pregnancy may facilitate lactogenesis II.

are related to adequate sleep rates. Studies of women found $36 \%$ of black or African American women versus $27 \%$ of white women sleep six hours or less per day. Thirty-three percent of women below poverty versus $25 \%$ of women with income 4-times the poverty level reported 6 hours or less of sleep per day (Schoenborn, Adams \& Peregoy 2013). Sleep quality in pregnant women was also found to be associated with race, ethnicity, age and socioeconomic status (Facco et al., 2017; Reid et al., 2017).

We recently completed a study that measured sleep quality and maternal mood using the Pittsburgh Sleep Quality Index (PSQI) and the Edinburgh Postnatal Depression Scale (EPDS) during gestational weeks 22 and 32 (Ahmed et al., 2018). Poorer maternal mood score was found to be associated with poorer sleep quality scores. Sleep scores were also found to be associated with work schedules. However, no significant association was found between self-reported sleep and blood glucose or development of gestational related disease. As part of the same study, sleep was objectively measured using wrist actigraphy during gestational weeks 22 and 32, as total sleep time is often over estimated when self-reported (Wilson, D.L., Fung, A., Walker, S.P. \& Barnes, M., 2013). The objective of this study was to analyze actigraphic data to determine if objectively measured sleep variables were associated with blood glucose levels, body mass index, development of gestational related disease, or work schedule. Moreover, the relationship between sleep duration, timing and continuity with DLII has not been studied. We hypothesized that short sleep duration and poorer sleep continuity during pregnancy is associated with DLII (Fu et al., 2015). Thus we also aimed to determine if there was a relationship between objectively measured sleep variables with DLII. 


\section{Methods}

\section{Design}

A prospective, longitudinal, one-group observational study was used as it suggests relationships, and reliable inferences. Thus, it allowed the analysis of sleep patterns of participants throughout pregnancy, and the analysis of relationships between sleep variables and the risk of gestational diseases and delayed lactogenesis II ( LoBiondo-Wood \& Haber, 2014). The study was approved by Institutional Review Boards (IRB) at Purdue University, Indiana University, and Eskenazi Health.

\section{Setting}

The study was conducted in Indianapolis, IN, an urban area in the Midwest, which is the sixteenth most populous city in the US. Indianapolis has an estimated population of 863,002 with the greater metropolitan area having a little over two million residents. The ethnic and racial mix of the population served by the prenatal clinic from which participants were recruited was approximately $35 \%$ Hispanic, $30 \%$ AfricanAmerican, 30\% Caucasian, and 5\% other. All data were collected from participants from August 2014 to October 2015.

\section{Sample}

Participants were recruited from an Indiana University affiliated prenatal clinic that has a mission to serve the underserved population. Inclusion criteria were primiparity, 18-40 years of age, expecting a single infant, and willingness to breastfeed. There were no exclusion criteria. Ninety-two women were recruited for the study with the assumption of $\sim 60 \%$ retention rate for a target of 50 women. Rationale for sample size was based on a priori power calculation. Data from a study by Herring et al. (2014) was used to calculate the power in detecting differences in sleep duration between women with and without hyperglycemia. A power of $89 \%$, with the alpha error rate set at $5 \%$, was found for a sample size of 50 .

\section{Measurements}

Data collected were discrete variables on participant demographics, work schedule, disease diagnosis, and self-scores of changes in breast fullness from time of parturition. Continuous variable data collected were blood glucose levels, body mass index (BMI), and actigraphic measures of rest-activity.

Demographic data (e.g. participant's age, education, ethnicity, race, occupation, and work schedule) were collected at study intake, using a 24-question survey. Consent was obtained from women to collect protected health information (PHI) from medical records, which included data on physiological variables (e.g. pre-pregnancy BMI, blood glucose levels after $50 \mathrm{~g}$ glucose challenge test and diagnosis of hypertension, preeclampsia, and GDM during pregnancy). The diagnosis of gestational related disease was defined according to the American Congress of Obstetrics and Gynecologists (ACOG) guidelines. The 50g glucose challenge test had been performed between 24-28 weeks pregnancy according to the ACOG guidelines (ACOG, 2018).

Actigraphic data were collected in 30-sec epochs and Actiware software (Version 5.04; Respironics, Inc.) algorithms were used to estimate sleep parameters. Researchers determined the nightly rest intervals by using a combination of event markers on actigrams set by the participant to indicate start and end of nightly rest, light exposure data collected by Actiwatch, and activity levels recorded on the actogram. Researchers verified nightly rest intervals against the time recorded in the participants' sleep logs (Patel et al., 2015).

Sleep variables measured were: (1) the night-by-night actigraphic estimates of sleep onset time (start sleep timeclock time of the first epoch scored as sleep in each rest interval), (2) end sleep time (clock time of the last epoch scored as sleep in each rest interval), (3) total sleep time (TST-number of minutes scored as sleep in each rest interval) and (4) sleep efficiency (SE-proportion of time from sleep onset to wake in each rest interval, scored as sleep, expressed as a percentage). The time in minutes of wakefulness after sleep onset during the night (WASO) and fragmentation index (FragI) were calculated. FragI-the sum of time awake related to total sleep time and the percentage of very short sleep periods $(\leq 15 \mathrm{~s})$ related to the total number of sleep periods. Therefore, this index represents restless sleep. Sleep measures were averaged across all available days of recording for each period (up to 7 days). Changes in the sleep variables across $24 \mathrm{hr}$ periods were assessed using the root mean square of successive difference (RMSSD; Burgess, Park, Wyatt, Rizvydeen \& Fogg, 2017; Straus, Drummond, Nappi, Jenkins \& Norman, 2015). RMSSD is a time-domain tool commonly used to assess heart rate variability (variability of heart rhythms), and by extension is used as a method to assess sleep (Bei, Wiley, Trinder \& Manber, 2016).

The self-report breast fullness survey was used, and is a validated-method that indirectly measures the timing of onset of lactogenesis II by maternal perception of changes in breast fullness in the post-partum (Perez-Escamilla \& Chapman, 2001). The survey asks women to report breast fullness on a 1 to 5 scale, (e.g.1 = no change since giving birth, $3=$ noticeably fuller, and $5=$ uncomfortably full, and 2 or 4 intermediate to respective order). DLII was defined as not reporting, at least, 'noticeably fuller' breasts (i.e. having a score of $<3$ ) by day 3 post-partum (Dewey et al., 2003; Nommsen-Rivers et al., 2010). This is criteria set forth by Chapman and Perez-Escamilla (2001) for determining if milk has come in (lactogenesis II has commenced). 
The infant feeding variable was measured at one-week postpartum. Participants completed a survey on how they had fed their infants since birth: exclusively breastfed, partially breastfed, or exclusively formula fed.

\section{Data collection}

The research team comprised two prenatal nurses, a project coordinator, and undergraduate and graduate students, who were trained by the project coordinator. Members of the research team (research assistants) administered the intake survey during the first prenatal visit following informed consent being received from the participants.

Participants were asked to wear a wrist actigraph (Actiwatch Spectrum, Philips Respironics, Andover, MA) on their non-dominant wrist and to keep a sleep diary for seven consecutive days during gestational weeks 22 and 32. Actigraphy is a validated method to measure objective sleep (Cellini, Buman, McDevitt, Ricker \& Mednick, 2013). Selection of gestational weeks 22 and 32 to measure sleep was aimed at capturing two distinct metabolic states of pregnancy, anabolic and catabolic, respectively (Meo \& Hassain, 2016). To collect actigaphy data, participants were contacted by a research assistant prior to and after gestational week 22 and 32 time-points by phone, SMS-message (text), and email, in order to schedule times for the pick-up and return of the actiwatch and sleep logs at the study site. At the time of pick-up, instructions were given on how to wear and operate the actiwatch, as well as how to fill in sleep logs.

To conduct the breast fullness survey, the research team was notified when participants were admitted to the hospital for delivery of their infant. A research assistant visited participants in the hospital within 36 hours of the birth of the infant, and conducted the first breast fullness survey after participants were given both verbal and written explanations of the concept of milk "coming in", which was developed by a lactation support provider. Thereafter, participants were contacted daily (by text message, phone call, or email) to complete the breast fullness survey until postpartum day 5 . A breastfeeding follow up survey was administered to participants at one week postpartum, either online, using Qualtrics, or by phone.

Protected health information was abstracted from medical charts by a research assistant after consent was obtained from participants. All data were entered into a HIPAAHealth Insurance Portability and Accountability Act of 1996 compliant, secured database (REDCap- Research Electronic Data Capture) that was administered by Indiana University (Harris, Taylor, Thielke, Gonzales \& Conde, 2009). The project coordinator reviewed data for accuracy. All consent forms and intake surveys were kept in the project coordinator's office in a locked file cabinet.

\section{Data analysis}

Statistical analysis was conducted using SPSS (IBM SPSS Version 24.0); a two-tailed $P$-value $\leq .05$ was considered significant. Wilcoxon signed-rank test (Kerby, 2014) was used to analyze whether there was a difference between gestational week 22 and 32 in each of the measured sleep variables (start and end sleep time, TST, SE, WASO, Fragl), and between groups with different BMI, gestation disease and DLII status. The effect size for Wilcoxon signed-rank test was calculated as the rank-biserial correlation, denoted $r$ (Kerby, 2014).

Simple linear regression analysis was used to determine if there was a significant bivariate relationship between sleep variables and blood glucose levels. Pearson Chisquare test was used to determine if the distribution of participants who experienced DLII varied by gestation disease incidence, BMI, blood glucose, or work schedule. Logistic multivariate regression models were used to assess the predictive value of sleep variables and health and life-style indicators (BMI, blood glucose level, and work schedule) at gestational week 22 for the development of gestational diseases or gestational week 32 for DLII. For variable selection, AIC (Akaike Information Criteria) was applied (Vrieze, 2012). A final analysis of sleep variables with time point gestational week and work schedules, limited to day workers and participants who were out-of-work, was conducted. Instead of only using the average of the sleep variable across all recorded days, all the characteristics of sleep of that point were used. For example, for each participant during gestational week 22 , seven measurements were used, if participant had seven days of data. For each participant, the measurements in different days were treated as repeated measures. Based on this, a linear regression model with repeated measures was tested using generalized estimating equations (GEE, Ming, 2014) for each of the sleep variables to explore the association between sleep variable and gestational time point, BMI, blood glucose level, and schedule (working day/out of work).

\section{Results}

\section{Characteristics of the sample}

Of the 92 women enrolled, 50 women completed gestational week 22, 46 completed gestational week 32 , and 42 women completed all aspects of the study. Changes in health status or other life events were reasons for approximately half of the study withdrawals $(n=26)$. The other half were withdrawn from the study by members of the research staff due to failure to comply with study protocol. Participants who completed all elements of the study had a median age of 23 years old, and two-thirds were black or African American $(n=27$; Table 1). Analysis of recruited, retained, and withdrawn 
Table I. Demographic and Health Characteristics of Study Sample $(n=42)$.

\begin{tabular}{lc}
\hline Characteristics & $n(\%)$ \\
\hline Race & \\
African American & $27(64)$ \\
White & $7(18)$ \\
More than one race, or other & $5(13)$ \\
Unknown/not reported & $3(5)$ \\
Ethnicity & \\
Hispanic & $6(14)$ \\
not Hispanic or Latino & $36(86)$ \\
Education & \\
Graduate degree & $6(14)$ \\
Bachelor degree & $5(11)$ \\
Associate degree & $3(7)$ \\
High school or GED & $26(64)$ \\
No high school or GED & $2(4)$ \\
Yearly household income & \\
Less than $\$ 10,000$ & $19(45)$ \\
$\geq \$ 10,000$, but $<\$ 25,000$ & $10(23)$ \\
$\geq \$ 25,000$, but $<\$ 50,000$ & $10(23)$ \\
$\geq \$ 50,000$ & $4(9)$ \\
Diagnosis & \\
Pre-eclampsia & $5(11)$ \\
GDM & $4(9)$ \\
Hypertension & $21(25)$ \\
Any gestational disease & $14(33)$ \\
BMl $>25$ & $20(48)$ \\
Hyperglycemia (blood glucose $\geq 130 \mathrm{mg} / \mathrm{dL})$ & $7(16)$ \\
\hline
\end{tabular}

Note. Data presented describe the demographics and rate of diagnosis among the $46 \%$ of participants ( $n=42)$ who completed all aspects of the study. The total sample size was $\mathrm{N}=92$.

"Any gestational disease is percent of population with any diagnosis of preeclampsia, hypertension, and/or gestational diabetes mellitus (GDM) therefore percent not additive across diagnosis, $\mathrm{BMI}=$ Body mass index.

populations found no difference in demographic variables among groups.

\section{Relationships Between Gestational Week, Sleep and Activity Variables}

The mean number of days of recorded actigraphic data were $6.5(0.8)$ days at gestational week 22 and $6.6(0.8)$ days at gestational week 32 , with minimum number of recorded days at 3.5 and 4 , respectively. There was no difference between mean total night sleep time for gestational week 22 (7.35 (1.18) hr/night) and gestational week 32 (7.13 (1.06) hr/night; Table 2). However, sleep efficiency was significantly decreased between gestational week 22 and gestational week 32, while WASO and FragI significantly increased, indicating that sleep continuity decreased between the second and third trimester. There were no significant differences in RMSSD, suggesting there was no change in night-to-night variability in the sleep measures between gestational time points.
Table 2. Comparison of Sleep Variables Measured by Wrist Actigraphy during Gestational Week 22 and $32^{\text {a }}$.

\begin{tabular}{|c|c|c|c|c|}
\hline & $\begin{array}{l}\text { Gestational } \\
\text { Week } 22\end{array}$ & $\begin{array}{l}\text { Gestational } \\
\text { Week } 32\end{array}$ & & \\
\hline & $n=50$ & $n=44$ & & \\
\hline & $M(S D)$ & $M(S D)$ & $r$ & $p$ \\
\hline Start sleep time & $00: 20(1.9)$ & $00: 20(1.9)$ & 0.07 & 0.67 \\
\hline End sleep time & $06: 48(3.6)$ & $07: 30(2.7)$ & 0.20 & 0.19 \\
\hline Total sleep time (min) & $441(71)$ & $424(62)$ & 0.14 & 0.39 \\
\hline Sleep efficiency $(\%)^{b}$ & $88(4)$ & $86(6.5)$ & 0.39 & 0.01 \\
\hline WASO (min) $)^{c}$ & $61(25)$ & $73(36)$ & 0.37 & 0.02 \\
\hline Fragl $^{d}$ & $21.6(6.2)$ & $24.9(8.4)$ & 0.49 & 0.001 \\
\hline
\end{tabular}

'based on Wilcoxon rank sum test

bSleep efficiency is the proportion of time from sleep onset to wake in each rest interval, scored as sleep, expressed as a percentage.

WASO is wake after sleep onset.

${ }^{d}$ Fragl is fragmentation index, which is the sum of time awake related to total sleep time and the percentage of very short sleep periods $(\leq 15$ s) related to the total number of sleep periods, and therefore is an index of restless sleep.

Table 3. Correlation of Blood Glucose Levels with Sleep Variables During Gestational Weeks 22 and 32.

\begin{tabular}{lccccc}
\hline & \multicolumn{2}{c}{ Gestational week 22 } & & \multicolumn{2}{c}{ Gestational week 32 } \\
\cline { 2 - 3 } \cline { 5 - 6 } & \multicolumn{2}{c}{$n=50$} & & \multicolumn{2}{c}{$n=44$} \\
\cline { 2 - 3 } \cline { 5 - 6 } mean & $r$ & $p$ & & $r$ & $p$ \\
\hline Start sleep time & -0.15 & 0.35 & & 0.10 & 0.53 \\
End sleep time & -0.02 & 0.90 & & -0.10 & 0.56 \\
Total sleep time & 0.01 & 0.95 & & -0.37 & 0.02 \\
Sleep efficiency & -0.13 & 0.41 & & -0.21 & 0.19 \\
WASO $^{b}$ & 0.13 & 0.41 & & 0.13 & 0.43 \\
Fraglc & 0.18 & 0.26 & & 0.42 & 0.01 \\
\hline
\end{tabular}

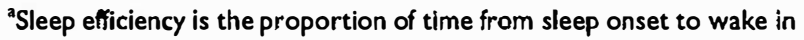
each rest interval, scored as sleep, expressed as a percentage.

'WASO is wake after sleep onset,

'Fragl is fragmentation index, which is the sum of time awake related to total sleep time and the percentage of very short sleep periods ( $\leq 15 \mathrm{~s})$ related to the total number of sleep periods, and therefore is an index of restless sleep.

\section{Relationship of Sleep and Activity With Health Indicators}

Approximately $16 \%(n=30)$ of the participants had hyperglycemia ( $\geq 130 \mathrm{mg} / \mathrm{dL}$ ). Linear regression analysis showed no significant relationship between actigraphic mean or RMSSD of sleep measures at gestational week 22 with blood glucose levels (Table 3). Blood glucose levels were negatively associated with total sleep duration (time) and positively associated with FragI at gestational week 32 .

Approximately $48 \%(n=24)$ of the participants had healthy pre-pregnancy BMI $(>18.5,<25), 4 \%(n=2)$ were underweight $(\mathrm{BMI}<18.5), 20 \%(n=10)$ were 
Table 4. Comparison of Sleep Variables Grouped by Diagnosis of Gestational Related Disease During 22 and 32 Gestational Weeks $(\mathrm{N}=45)$.

\begin{tabular}{|c|c|c|c|c|c|c|c|c|}
\hline & \multicolumn{2}{|c|}{ Gestational Weeks 22} & \multirow[b]{4}{*}{$r$} & \multirow[b]{4}{*}{$p$} & \multicolumn{2}{|c|}{ Gestational weeks 32} & \multirow[b]{4}{*}{$r$} & \multirow[b]{4}{*}{$p$} \\
\hline & No disease & Disease & & & No disease & Disease & & \\
\hline & $n=30$ & $n=15$ & & & $n=29$ & $n=15$ & & \\
\hline & $M(S D)$ & $M(S D)$ & & & $M(S D)$ & $M(S D)$ & & \\
\hline Start sleep time & $00: 14(2.01)$ & $00: 27(2.20)$ & 0.09 & 0.57 & $00: 20(1.42)$ & $23: 57(1.70)$ & 0.10 & 0.50 \\
\hline End sleep time & $06: 48(3.5)$ & $06: 55(3.6)$ & 0.05 & 0.75 & $07: 41(2.66)$ & $07: 19(2.87)$ & 0.07 & 0.64 \\
\hline Total sleep time (min) & $434.5(63.9)$ & $433.7(6 \mid .2)$ & 0.07 & 0.66 & $434.0(53.8)$ & $412.2(74.7)$ & 0.12 & 0.43 \\
\hline Sleep Efficacy $(\%)^{\mathrm{a}}$ & $89.12(4.29)$ & $87.69(3.8)$ & 0.15 & 0.31 & $87.62(4.33)$ & $82.58(9.25)$ & 0.27 & 0.07 \\
\hline WASO $(\min )^{b}$ & $54.67(21.7)$ & $64.70(30.4)$ & 0.12 & 0.43 & $63.10(23.5)$ & $90.60(49.3)$ & 0.26 & 0.08 \\
\hline Fragl $^{c}$ & $20.13(6.66)$ & $22.39(4.28)$ & 0.16 & 0.30 & $22.55(6.27)$ & $29.81(10.6)$ & 0.34 & 0.02 \\
\hline
\end{tabular}

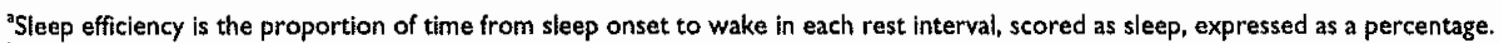

bWASO is wake after sleep onset.

'Fragl is fragmentation index, which is the sum of time awake related to total sleep time and the percentage of very short sleep periods ( $\leq 15 \mathrm{~s}$ ) related to the total number of sleep periods, and therefore is an index of restless sleep.

overweight $(\mathrm{BMI}>25,<30)$, and $28 \%(n=14)$ were obese (BMI > 30). Mean blood glucose levels were significantly lower in participants with BMI $<25(96.5 \mathrm{mg} / \mathrm{dL}$ (8.0) than women with BMI $\geq 25(115.0 \mathrm{mg} / \mathrm{dL}$ (31.5). Mean sleep metrics were not different between BMI groups (Supplemental Table S1).

The rate of diagnosis of GDM, pre-eclampsia, and hypertension were $11 \%(n=5) 13 \%(n=6)$, and $27 \%(n=12)$, respectively. The sample size was too small to study the potential relationship of each disease with sleep variables, thus analyses were conducted to examine the development of any gestational related disease (GDM, pre-eclampsia, and hypertension), which there was in $33 \%(n=15)$ of the population. Women with gestational disease had significantly greater sleep fragmentation (FragI) at gestational week 32 (Table 4).

\section{Relationship of Health and Sleep Variables with Timing of Lactogenesis II}

Participants showed progressive changes in self-reported breast fullness in the first few days post-partum (Figure 1). By day three, $69 \%(n=29)$ of participants reported breasts were "noticeably fuller" or more (a score of 3 or more). Thus, approximately $31 \%(n=15)$ of participants experienced DLII. Blood glucose levels were not different between participants who experienced DLII from those who did not. The rate of overweight/obesity in participants who experienced DLII $(46 \%, n=6)$ was similar to those that did not $(44 \%, n=13)$. Approximately $33 \%(n=5)$ of the participants who experienced DLII had developed a gestational related disease; this rate of gestational disease was not different from the $31 \%(n=9)$ of participants who did not experience DLII.

Rate of cesarean delivery, length of labor, infant birthweight, and gestational age at birth were not significantly different between DLII and non DLII groups (see Supplemental Materials Table S2). Although frequency of suckling was not measured in this study, we were able to evaluate first feeding and exclusivity at one week. In particular, first feeding data were available in medical charts from the majority of the study participants $(n=39)$; all participants were reported to have breastfed their infant at first feeding. At one week postpartum, participants were asked how they had fed their infant since birth. Although there was a difference in rates of exclusive breastfeeding among participants who experienced DLII $(27 \% ; n=4)$ versus those who did not $(42 \% ; n=12)$, the difference was not statistically significant at this sample size $(p=$ 0.22 ). One participant in each group was exclusively feeding infant formula; all others were feeding both breastmilk and formula.

Participants who experienced DLII had significantly lower sleep efficiency at gestational week 32 compared to participants who did not (Table 5). There was a tendency for lower sleep efficiency in the DLII group at gestational week 22 (Table 5). WASO was significantly higher in participants who experienced DLII compared to those who did not at gestational week 32 (Figure 2A). There was also a tendency for a difference in WASO between the groups at gestational week 22. Mean sleep fragmentation index at gestational week 32 was greater in participants who experienced DLII. Night to night variation in start and end of sleep time (Figure 2B), total sleep time, SE, and WASO at gestational week 32 was greater in participants who experienced DLII, as was indicated by greater RMSSD values than in those who did not. RMSSD of sleep fragmentation index at gestational week 22 was significantly greater in participants who experienced DLII than those who did not (Table 5).

A multiple logistic regression model was used to evaluate the relationship between sleep variables and DLII. For the 


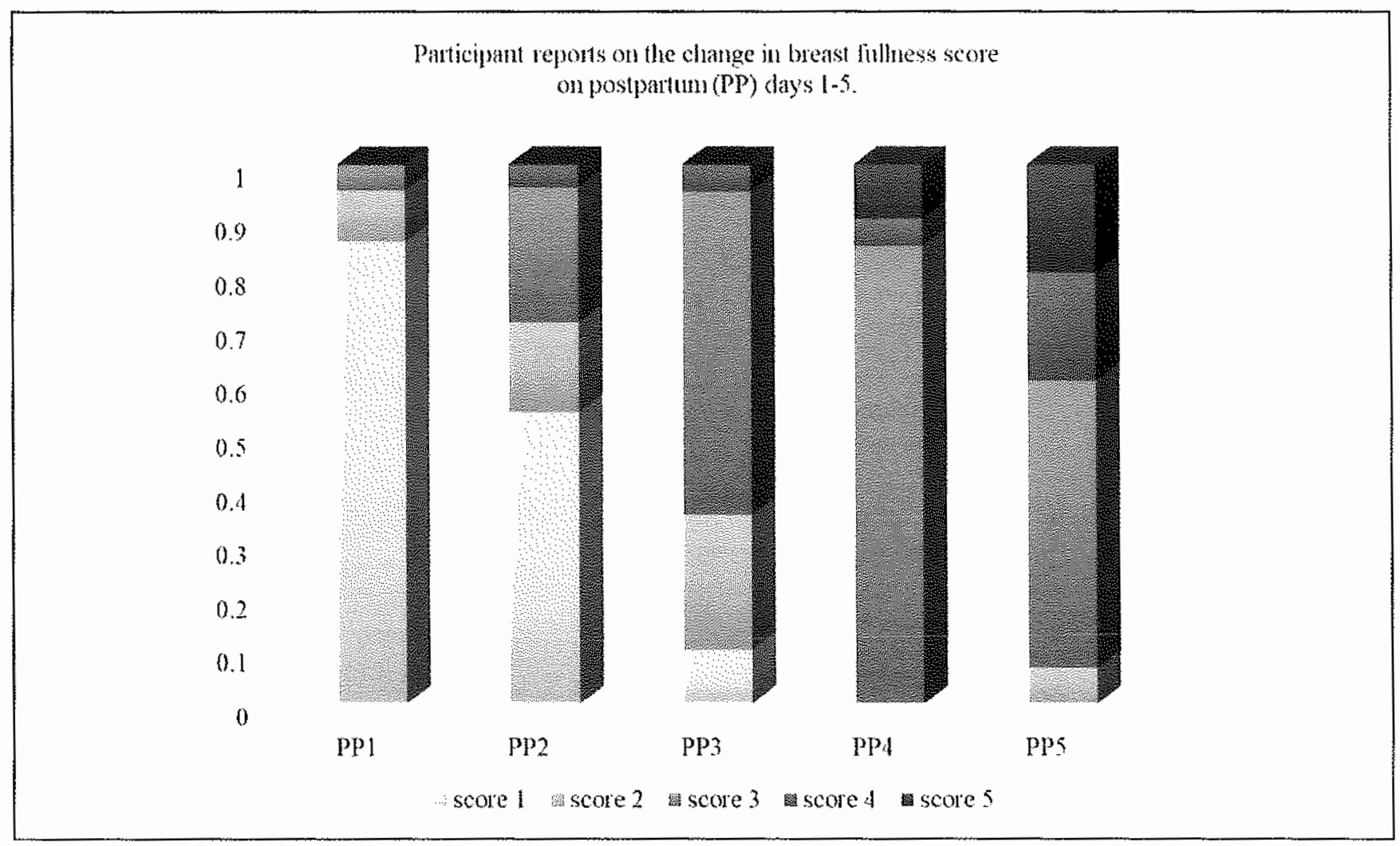

Figure I. Proportion of women reporting breast fullness score I-5, on postpartum days one through five. Score of $1=$ no change since giving birth, $3=$ noticeably fuller, and $5=$ uncomfortably full. A score of $<3$ on postpartum day 3 was considered DL.ll.

Table 5. Comparison of Sleep Variables During 22 and 32 Gestational Weeks Grouped by Experience of Delayed Lactogenesis II (DLII) $(N=44)$.

\begin{tabular}{|c|c|c|c|c|c|c|c|c|}
\hline & \multicolumn{2}{|c|}{ Gestational Week 22} & \multirow[b]{4}{*}{$r$} & \multirow[b]{4}{*}{$p$} & \multicolumn{2}{|c|}{ Gestational Week 32} & \multirow[b]{4}{*}{$r$} & \multirow[b]{4}{*}{$p$} \\
\hline & No DLII & DLII & & & No DLIII & DLII & & \\
\hline & $n=29$ & $n=15$ & & & $n=29$ & $n=15$ & & \\
\hline & $M(S D)$ & $M(S D)$ & & & $M(S D)$ & $M(S D)$ & & \\
\hline Start sleep time & $00: 14(1.53)$ & $00: 13(2.81)$ & 0.05 & 0.75 & $00: 27(1.43)$ & $23: 54(1.61)$ & 0.23 & 0.13 \\
\hline End sleep time & $07: 02(2.86)$ & $06: 28(4.62)$ & 0.06 & 0.71 & $08: 19(1.23)$ & $06: 14(4.08)$ & 0.30 & 0.02 \\
\hline Total sleep time (min) & $434.7(59.0)$ & $433.4(70.0)$ & 0.03 & 0.85 & $438.0(54.1)$ & $416.5(60.8)$ & 0.15 & 0.34 \\
\hline Sleep efficiency (\%)a & $89.51(3.85)$ & $87.18(4.44)$ & 0.28 & 0.06 & $88.14(4.31)$ & $83.56(6.04)$ & 0.35 & 0.02 \\
\hline WASO (min)b & $52.49(20.3)$ & $67.28(29.6)$ & 0.27 & 0.07 & $61.10(25.4)$ & $86.01(38.1)$ & 0.36 & 0.02 \\
\hline \multirow[t]{2}{*}{ Fraglc } & $19.70(5.37)$ & $22.87(6.87)$ & 0.22 & 0.15 & $22.50(5.69)$ & $27.42(8.21)$ & 0.31 & 0.04 \\
\hline & $\operatorname{RMSSD}^{d}(\mathrm{SD})$ & RMSSD (SD) & $r$ & $p$ & RMSSD (SD) & RMSSD (SD) & $r$ & $p$ \\
\hline Start sleep time & $1.71(0.93)$ & $2.15(1.68)$ & 0.10 & 0.51 & $1.47(0.76)$ & $2.13(0.93)$ & 0.36 & 0.02 \\
\hline End sleep time & $1.63(1.41)$ & $1.91(1.09)$ & 0.23 & 0.13 & $1.37(0.59)$ & $2.59(1.23)$ & 0.51 & 0.0003 \\
\hline Total sleep time $(\mathrm{min})$ & $|21|.(67.7)$ & $136.2(74.8)$ & 0.13 & 0.42 & $94.61(41.7)$ & $152.1(54.4)$ & 0.47 & 0.001 \\
\hline Sleep efficiency $(\%)$ & $4.40(2.49)$ & $6.17(3.15)$ & 0.27 & 0.07 & $4.74(4.29)$ & $7.61(6.03)$ & 0.32 & 0.03 \\
\hline WASO (min) & $30.65(18.5)$ & $44.62(30.3)$ & 0.26 & 0.09 & $33.38(34.4)$ & $62.29(38.6)$ & 0.46 & 0.002 \\
\hline Fragl & $7.49(3.62)$ & $11.25(4.38)$ & 0.39 & 0.008 & $8.91(5.38)$ & $10.42(5.28)$ & 0.14 & 0.36 \\
\hline
\end{tabular}

aSleep efficiency is the proportion of time from sleep onset to wake in each rest interval, scored as sleep, expressed as a percentage.

WASO is wake after sleep onset.

'Fragl is fragmentation index, which is the sum of time awake related to total sleep time and the percentage of very short sleep periods ( $\leq 15 \mathrm{~s}$ ) related to the total number of sleep periods, and ther efore is an index of restless sleep

${ }^{\delta} R M S S D$ is root mean square of successive difference. 


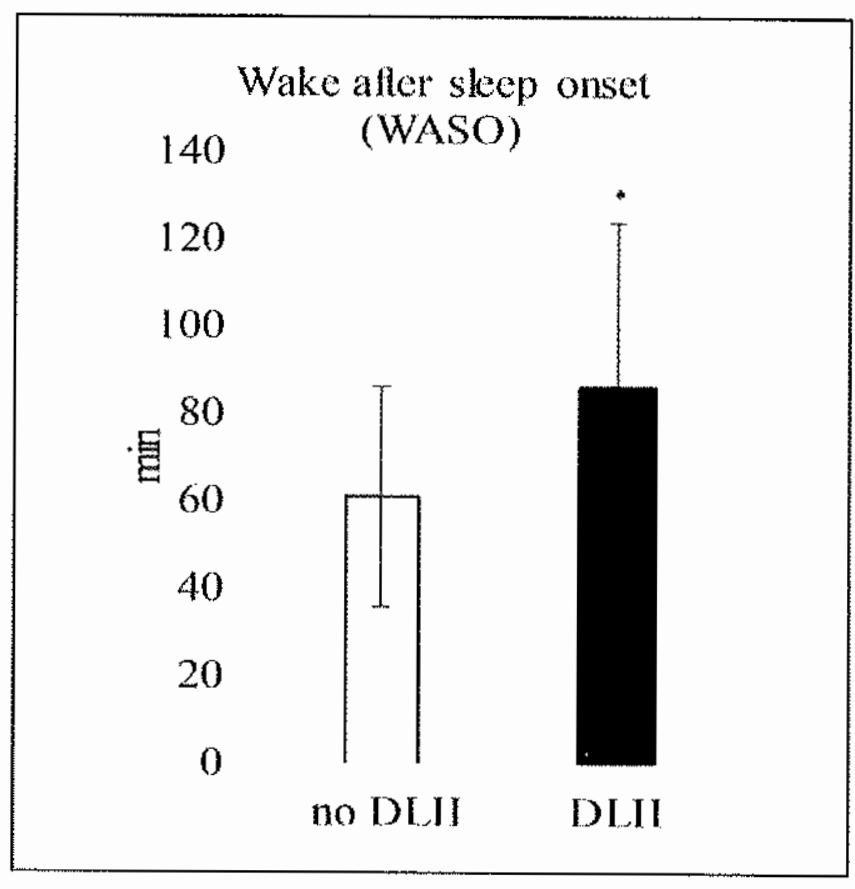

Figure 2A. WASO During Gestation Week 32 (G32) in Study Participants who did not (no DLII) and did (DLII) Experience Delayed Onset of Lactogenesis II. Values are mean or RMSSD \pm SD. (* indicates a difference at $\mathrm{P}<0.05$ ).

full model sleep variables from gestational week 32 along with BMI, blood glucose levels and gestational disease were considered. This was followed by variable selection with the smallest AIC to develop the final model. The final model indicated sleep efficiency and the RMSSD of total sleep time were predictors for the model with a goodness-of-fit test $p$ $<0.01$. The model showed that DLII was significantly related to sleep efficiency and the RMSSD of total sleep time during gestational week 32 .

\section{Relationship of Work Schedule to Sleep Variables}

Information regarding individual's work schedules was collected from 45 women by survey at study intake. The relationship between work schedules was analyzed to determine if life-style factors were related to the differences among BMI, disease, DLII groups, and sleep variables. Approximately $48 \%(n=22)$ of women reported working day shifts, $24 \%(n=11)$ were out of work, and $26 \%(n=12)$ worked nights or shift-work. Work schedules had a significant relationship with blood glucose, with glucose levels significantly higher in women who worked nights or shift work (120.5 (35.2) $\mathrm{mg} / \mathrm{dL}$ ) schedules versus those who worked day shifts $(97.25(19.7) \mathrm{mg} / \mathrm{dL} ; p<.05)$. There was no difference in mean pre-pregnancy BMI between groups. Frequency of gestational-related disease between groups was similar, $33 \%(n=7), 27 \%(n=3)$, and $33 \%(n=4)$, as was the frequency of women with DLII $38 \%(n=8)$,

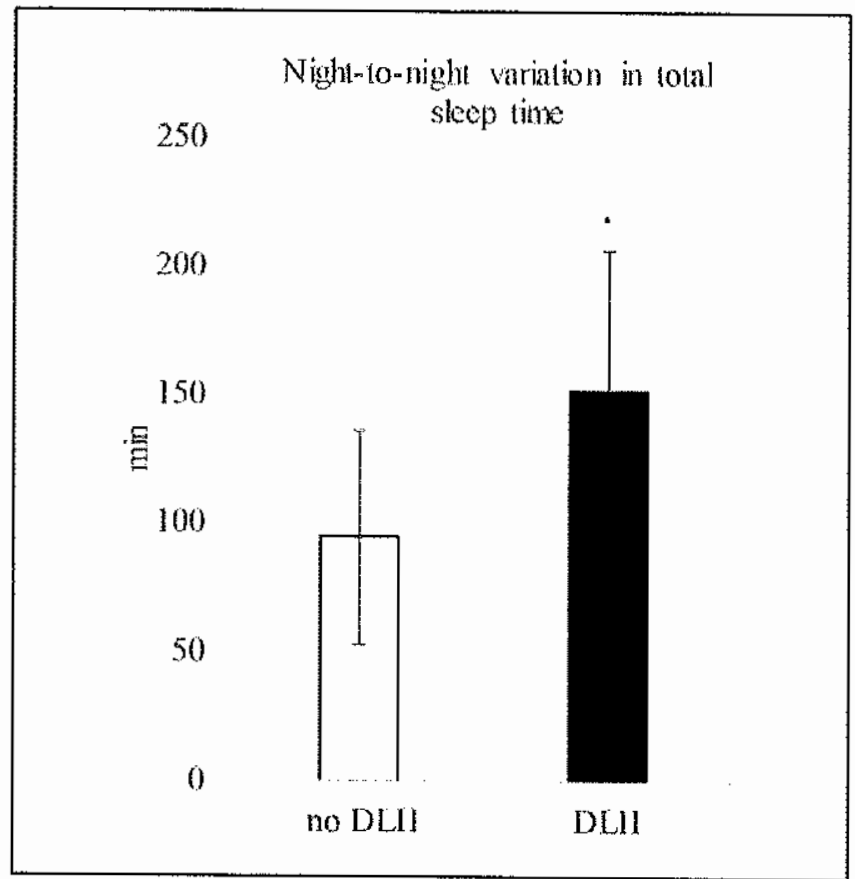

Figure 2B. Night-to-Night Variation (RMSSD) of Total SleepTime During Gestation Week 32 (G32) in Study Participants who did not (no DLII) and did (DLII) Experience Delayed Onset of Lactogenesis II. Values are mean or RMSSD \pm SD. (* indicates a difference at $\mathrm{P}<0.05$ ).

$27 \%(n=3)$, and $33 \%(n=4)$, respectively by work schedule (day-shift, out-of-work, night/rotating shift). The Pearson Chi-square test found no significant relationship between disease or DLII and work schedule.

Work schedule was not significantly related to any of the sleep variables (Supplemental Table S3), although there was a weak signal for a difference in average start and end of sleep time during gestational week 32 . However, because night-shift work is associated with changes in hormones and metabolites and health, the relationships between sleep and health indicators, including DLII, were reexamined with the night/shift workers removed to determine if that affected the results. Reanalysis found a weak signal for an increase in FragI between gestational week 22 and 32 . Blood glucose levels had a negative relationship with sleep efficiency during both gestational week 22 and 32 , as well as total sleep time during gestational week 32 . In addition, blood glucose was positively associated with Frag1 during gestational week 32. There was no relationship between development of gestational related disease and sleep metrics at gestational week 22 or 32 .

Repeated measure simple linear regression models using GEE revealed that gestational time point significantly explained differences in SE, WASO, and FragI. Blood glucose level had a significant impact on SE, WASO, and FragI. Moreover, work status had a significant association with start sleep time. Women out of work had a later start sleep time than daytime workers (diff $=1$ hour, $p=.05$ ). As blood 
glucose level increased by one unit, sleep efficiency decreased by $0.10 \%(p<.05)$, and sleep efficiency during gestational week 32 was worse than 22 (diff $=1.5 \%, p<$ $.05)$. WASO increased by $0.48(p=.04)$ and Fragl increased by $0.12(p=.054)$ as blood glucose levels increased by one unit. Furthermore, WASO and FragI both significantly increased from gestational week 22 to $32(p<.05)$.

Reanalysis of the relationship of DLII with sleep characteristics in the subset of women (day shift and out-of-work) found RMSSD of sleep start (1.42 (8.2) and $1.99(0.57) ; p=$ $0.02)$ and end (1.41 (0.58) and $2.41(0.97) ; p=.004)$ time during gestational week 32 to be significantly different between women who did not experience DLII, and those who did, respectively. RMSSD of total sleep time (90.62 (43.5) and $151.17(51.9) ; p=.004)$ and WASO (32.35 (39.3) and 46.01 (7.25); $p=.01$ ) during gestational week 32 were significantly different between women who did not experience DLII, and those who did, respectively. A final simple logistic model was used to test associations and DLII was found to be related to the RMSSD of total sleep time during gestational week $32(p=.02)$.

\section{Discussion}

Our main finding was that lower sleep efficiency and greater variation in nightly sleep duration in the third trimester was associated with delayed lactogenesis II. This finding supports our hypothesis and, to our knowledge, is novel. Here we discuss study findings in relation to consistency to previously published data on sleep during pregnancy and maternal physiology. Also discussed is the implication of findings for the clinical management of breastfeeding women and the limitations of the study.

Relative to non-pregnant women, pregnant women experience increased sleep fragmentation (Gay, Richoux, Beebe \& Lee, 2017), lower sleep efficiency, poorer subjective sleep quality, and higher daytime sleepiness (Berndt, Diekelmann, Alexander, Pustal \& Kirschbaum, 2014). However, pregnant women slept longer in total than non-pregnant controls. We found that between the second and third trimester, although total sleep time did not change, sleep quality deteriorated, with sleep efficiency decreasing and Fragl increasing between gestational week 22 and 32, which is consistent with findings of Tsai et al. (2016).

Similar to previous studies of pregnant women (Herring et al., 2014; Reutrakul et al., 2011), we found an inverse association between nighttime sleep duration and blood glucose. Blood glucose level was inversely related to sleep efficiency, and positively related to sleep fragmentation index during gestational week 32 . Moreover, higher blood glucose was found in participants who worked night/shift schedules versus those who worked during the daytime. This is consistent with findings of a systematic review that concluded shift-work was related to the development of hyperglycemia and type II diabetes (Knutsson \& Kempe, 2014).
Obesity has been associated with greater variability in time in bed and sleep duration in non-pregnant women (Reid et al., 2017). In pregnant women higher WASO was related to greater BMI (Haney et al., 2014), and excess gestational weight gain was associated with poorer perceived sleep quality, but was unrelated to objective measures of sleep duration and disruption (Gay, Richoux, Beebe \& Lee, 2017). In our sample, there was only a weak signal for an association of overweight/obesity with more fragmented sleep during the third trimester. We found that gestational time point significantly explained differences in sleep efficiency, WASO, and FragI, suggesting that factors associated with pregnancy and not BMI were driving differences in sleep metrics.

Self-reported and objectively measured shorter sleep duration have been associated with development of GDM, hypertension, and preeclampsia (Cai et al., 2017; Facco et al., 2017; Qiu, Enquobahrie, Frederick, Abetew \& Williams, 2010; Rawal, Hinkle, Zhu, Albert \& Zhang, 2017; Williams et al., 2010). We did not find a relationship between total nighttime sleep and development of gestational-related disease. However, greater sleep fragmentation and a tendency for poorer sleep efficiency during gestational week 32 was found in participants who developed a gestational related disease.

Participants who experienced DLII had significantly lower sleep efficiency, and significantly higher WASO and sleep fragmentation during gestational week 32 . During gestational week 22, there was also a tendency for lower sleep efficiency and higher WASO in the DLII group. Night-to-night variation in start and end of sleep time, total sleep time, sleep efficiency, and WASO at gestational week 32 was greater in participants who experienced DLII. DLII was significantly related to average sleep efficiency and the RMSSD of total sleep time during gestational week 32 , supporting the finding that women with better sleep efficiency and more stable night-to-night sleep duration will have a lower risk of DLII.

To ensure findings were not due to the shift-workers in the respective samples, analyses were rerun after removal of this population. The relationship of DLII with sleep characteristics remained after the shift-workers were removed. Specifically, the RMSSD of total sleep duration and sleep start and end time during G32 were significantly greater in participants who experienced DLII.

Sleep is cooperatively regulated by homeostatic and circadian factors, with reciprocal regulation among the circadian timing system and sleep-wake cycle. Reciprocal regulation also occurs among circadian and metabolic systems, and thus it is not surprising that study findings support a mechanistic link between inadequate sleep, circadian disruption, and the development of metabolic disease (Poggiogalle, Jamshed \& Peterson, 2018). Changes in hormones and metabolism occur in the periparturient period to divert energy and substrates to the mammary glands to initiate lactogenesis II (Fu et al., 2015). Poorer sleep continuity, as indicated by lower sleep efficiency and greater WASO and FragI, may be associated with DLII because it alters the 
metabolic and hormonal environment needed to initiate lactogenesis II.

Further studies are needed to conclusively link sleep and circadian disruption with metabolic and hormonal disturbances that affect lactogenesis II. Also needed are intervention studies aimed at determining the impact of raising a woman's awareness of sleep, eating, and exercise patterns through diaries and self-monitoring, and educating women about good sleep habits and the consequences of sleep deprivation, and how exposure to light at night during gestation affects the timing of lactogenesis II. Studies that examine whether sleep and circadian disruption affect milk production during an established lactation are also need. These may be particularly important for developing breastfeeding management guidelines for women returning to shift-work after the birth of their infant.

The relationship of DLII with higher night-to-night variation in total sleep time, even after the shift-workers were removed from analyses, suggest that self-imposed (versus work imposed) social jet lag may be an issue in the study population. More than half the population studied was less than 23 years old and had an annual income of less than $\$ 25,000$. Two-thirds of participants were African-American women, with the highest education level being high school graduate. Sleep quality in pregnant and non-pregnant women varies with demographics. In studies of pregnant women, younger African-American women with a lower socioeconomic status were associated with relatively poorer sleep metrics (Facco et al., 2017; Reid et al., 2017). Intervention studies aimed at these populations are thus particularly warranted.

\section{Limitations}

The primary limitation of our study was the small sample size, with $54 \%$ of recruited population lost to follow-up. Therefore, the interpretation of findings is limited. However, findings support our hypothesis, justifying a study of a larger population which will enable analysis of the relationship of sleep, gestational related disease, and timing of lactogenesis II with covariates and confounders. Secondly, sleep apnea was not assessed in the study and may be driving some of the sleep discontinuity found, and will require further studies. In addition, despite the validation of actigraphy in objectively measuring sleep (Cellini et al., 2013), limitations exist for estimating some sleep/awake parameters relative to polysomnography. Overall actigraphic sensitivity and accuracy are high relative to polysomnography, but specificity is low (Marino et al., 2013). Furthermore, although meta-analysis found the breast fullness survey adequate for determining the timing of lactogenesis II (PerezEscamilla \& Chapman, 2001), using a biomarker such as measuring milk and serum lactose or citrate levels, or weighing breasts (Murase, Wagner, J. Chantry, Dewey \& Nommsen-Rivers, 2017; Panwara, Kasem, Wipada, Maysita
\& Pawin, 2016; Rebecca et al., 2018), would strengthen the approach to determining DLII. Moreover, data available from medical charts on labor length and the timing of firstfeeding was sparse and varied by clinician. Active collection by research teams of data needed for a comprehensive assessment of the factors that affect lactogenesis II, rather than relying on abstracting information from medical charts, would increase the efficacy of the approach. Finally, there was lack of agreement between findings presented here and our analysis of self-reported sleep quality in the same population of pregnant women, which found no significant association between sleep scores during gestational week 22 and 32 and blood glucose levels nor gestational related diseases (Ahmed et al., 2018). Lack of agreement between the methods may reflect that self-reports of sleep made by both pregnant and non-pregnant women tend to be unreliable relative to objectively measured sleep, with women overestimating total sleep time and underestimating sleep latency (Wilson, D.L., Fung, A., Walker, S.P. \& Barnes, M., 2013).

\section{Conclusion}

Women who experienced DLII had significantly greater variations in start sleep time and lower sleep efficiency in the third trimester. Therefore, interventions that target sleep restoration might improve breastfeeding adequacy by decreasing the risk of DLII, which has been shown to contribute to early formula supplementation and breastfeeding cessation.

\section{Declaration of Conflicting Interests}

The authors declared no potential conflicts of interest with respect to the research, authorship, and/or publication of this article.

\section{Funding}

The authors disclosed receipt of the following financial support for the research, authorship, and/or publication of this article: AgSEED 2014 Grant- Agricultural Research and Extension Leading to Economic Development in Indiana Agriculture and Rural Communities.

\section{Supplemental Material}

Supplemental material for this article is available online.

\section{ORCID ID}

Theresa Casey https://orcid.org/0000-0002-8835-3550

\section{References}

Ahmed, A., Hui, S., Crodian, J., Plaut, K., Haas, D., Zhang, L., \& Casey, T. (2018). Relationship between sleep quality, depression symptoms, and blood glucose in pregnant women. Western Journal of Nursing Research. doi: 10.1177/0193945918809714

American Congress of Obstetrics and Gynecologists (ACOG) Committee on practice bulletins, (2018). Gestational diabetes mellitus. ACOGPractice BulletinNo. 190. Obstetrics and Gynecology, 13l(2):e49-e64. doi: 10.1097/AOG.0000000000002501 
Bei, B., Wiley, J. F., Trinder, J., \& Manber, R. (2016). Beyond the mean: A systematic review on the correlates of daily intraindividual variability of sleep/wake patterns. Sleep Medicine Review, 28, 108-124. doi: 10.1016/j.smrv.2015.06.003

Berndt, C., Diekelmann, S., Alexander, N., Pustal, A., \& Kirschbaum, C. (2014). Sleep fragmentation and false memories during pregnancy and motherhood. Behavioral Brain Research, 266, 52-57. doi: 10.1016/j.bbr.2014.02.030

Brownell, E., Howard, C.R., Lawrence, R.A., \& Dozier, A.M. (2012). Delayed onset lactogenesis II predicts the cessation of any or exclusive breastfeeding. The Journal of Pediatrics, 161(4), 608614. doi: http://dx.doi.org/10.1016/j.jpeds.2012,03.035

Burgess, H. J., Park, M., Wyatt, J. K., Rizvydeen, M., \& Fogg, L. F. (2017). Sleep and circadian variability in people with delayed sleep-wake phase disorder versus healthy controls. Sleep Medicine, 34, 33-39. doi: 10.1016/j.sleep.2017.02.019

Cai, S., Tan, S., Gluckman, P. D., Godfrey, K. M., Saw, S. M., Teoh, O. H., \& Gooley, J. J. (2017). Sleep quality and nocturnal sleep duration in pregnancy and risk of gestational diabetes mellitus. Sleep, 40(2). doi: 10.1093/sleep/zsw058

Cellini, N., Buman, M. P., McDevitt, E. A., Ricker, A. A., \& Mednick, S. C. (2013). Direct comparison of two actigraphy devices with polysomnographically recorded naps in healthy young adults. Chronobiology International, 30(5), 691-698. doi: $10.3109 / 07420528.2013 .782312$

Chapman, D. J., \& Perez-Escamilla, R. (2000). Maternal perception of the onset of lactation is a valid, public health indicator of lactogenesis stage II. The Journal of Nutrition, 130(12), 29722980.

Dewey, K.G., Nommsen-Rivers, L.A., Heinig, M.J., \& Cohen, R.J. (2003). Risk factors for suboptimal infant breastfeeding behavior, delayed onset of lactation, and excess neonatal weight loss. Pediatrics, 112, 607-619.

Facco, F. L., Grobman, W. A., Kramer, J., Ho, K. H., \& Zee, P. C. (2010). Self-reported short sleep duration and frequent snoring in pregnancy: Impact on glucose metabolism. American Journal of Obstetrics and Gynecology, 203(2), 142 e141-145. doi: $10.1016 /$ j.ajog.2010.03.041

Facco, F. L., Grobman, W. A., Reid, K. J., Parker, C. B., Hunter, S. M., Silver, R. M., \& Zee, P. C. (2017). Objectively measured short sleep duration and later sleep midpoint in pregnancy are associated with a higher risk of gestational diabetes. American Journal of Obstetrics and Gynecology., 217(4), 447 e441-447 e413. doi: 10.1016/j.ajog.2017.05.066

Fu, M., Zhang, L., Ahmed, A., Plaut, K., Haas, D. M., Szucs, K., \& Casey, T.M. (2015). Does circadian disruption play a role in the metabolic-hormonal link to delayed lactogenesis II? Frontier Nutrition, 2. doi: 10.3389/fnut.2015.00004

Gay, C. L., Richoux, S. E., Beebe, K. R., \& Lee, K. A. (2017). Sleep disruption and duration in late pregnancy is associated with excess gestational weight gain among overweight and obese women. Birth, 44(2), 173-180. doi: 10.1111/birt.12277

Haney, A., Buysse, D. J., Rosario, B. L., Chen, Y. F., \& Okun, M. L. (2014). Sleep disturbance and cardiometabolic risk factors in early pregnancy: a preliminary study. Sleep Medicine, 15(4), 444-450. doi: 10.1016/j.sleep.2014.01.003

Harris, P.A., Taylor, R., Thielke, R., Payne, J., Gonzalez, N., \& Conde, J.G. (2009) Research electronic data capture (REDCap) - A metadata-driven methodology and workflow process for providing translational research informatics support. Journal of Biomedical Information, 42(2):377-81

Herring, S. J., Nelson, D. B., Pien, G. W., Homko, C., Goetzl, L. M., Davey, A., \& Foster, G. D. (2014). Objectively measured sleep duration and hyperglycemia in pregnancy. Sleep Medicine, $15(1)$, 51-55. doi: 10.1016/j.sleep.2013.07.018

Hoban, R., Patel A. L., Medina Poeliniz, C., Lai, C. T., Janes, J, Geddes, D., \& Meier, P. P. (2018). Human milk biomarkers of secretory activation in breast pump-dependent mothers of premature infants. Breastfeeding Medicine, 13(5), 352-360. doi: $10.1089 / \mathrm{bfm} .2017 .0183$

IBM Corporation. (2016). IBM SPSS Statistics for Windows, Version 24.0. Armonk, NY: IBM Corporation.

Izci, B., Jackson, B., Ratcliffe, S. A., Pack, A.I., \& Pien, G.W. (2013). Sleep-disordered breathing and daytime napping are associated with maternal hyperglycemia. Sleep \& Breathing, 1-10. doi: $10.1007 / \mathrm{s} 11325-013-0809-4$

Kerby Dave, S. (2014). The simple difference formula: An approach to teaching nonparametric correlation., Comprehensive Psychology, 3, doi: $10.2466 / 11$,IT.3.1

Knutsson, A., \& Kempe, A. (2014). Shift work and diabetes - A systematic review. Chronobiology International,31(10), 11461151. doi: $10.3109 / 07420528.2014 .957308$

Lawrence, R. A., \& Lawrence, R. M. (2016). Physiology of lactation. Breastfeeding a guide for the medical profession. 8th ed. Philadelphia: Elsevier; p. 56-63. 28.

LoBiondo-Wood, G., \& Haber, J. (2014). Nursing research: Methods and critical appraisal for evidence-based practice $\left(8^{\text {th }}\right.$ ed.). London, Mosby.

Matias, S. L., Nommsen-Rivers, L. A., Creed-Kanashiro, H., \& Dewey, K. G. (2010). Risk factors for early lactation problems among Peruvian primiparous mothers. Maternal \& Child Nutrition, 6(2), 120-133. doi: 10.1111/j.1740-8709. 2009.00195.x

Marino, M., Li, Y., Rueschman, M.N., Winkelman, J.W., Ellenbogen, J.M., Solet, J.M., Dulin, H., Berkman, L.F., \& Buxton, O.M. (2013). Measuring sleep: accuracy, sensitivity, and specificity of wrist actigraphy compared to polysomnography. Sleep. 36, 1747-55. doi.org/10.5665/sleep. 3142

Meo, S. A., \& Hassain, A. (2016). Metabolic physiology in pregnancy. Journal of Pakistan Medical Association, 66(9 Suppl 1), S8-S10.

Ming, W. (2014) Generalized estimating equations in longitudinal data analysis: A review and recent developments. Advances in Statistics (Vol. 2014). doi.org/10.1155/2014/303728.

Morceli, G., Franca, E. L., Magalhaes, V. B., Damasceno, D. C., Calderon, I. M., \& Honorio-Franca, A. C. (2011). Diabetes induced immunological and biochemical changes in human colostrum. Acta Paediatrica, 100(4), 550-556. doi: 10.1111/j.1651-2227.2010.02070.x

Murase, Masahiko, Wagner, Erin A., Caroline, J. Chantry, Dewey, Kathryn G., \& Nommsen-Rivers, Laurie A. (2017). The relation between breast milk sodium to potassium ratio and maternal report of a milk supply concern. The Journal of Pediatrics, 181, 294-297.e293. doi: 10.1016/j.jpeds.2016.10.044

Nommsen-Rivers, L. A. (2016). Does insulin explain the relationship between maternal obesity and poor lactation outcomes? An overview of the literature. Advances in Nutrition, $7(2), 407-414$. 
Nommsen-Rivers, L. A., Chantry, C. J., Peerson, J. M., Cohen, R. J., \& Dewey, K. G. (2010). Delayed onset of lactogenesis among first-time mothers is related to maternal obesity and factors associated with ineffective breastfeeding. Journal of Clinical Nutrition. 92(3), 574-584. doi: 10.3945/ ajen.2010.29192

Nommsen-Rivers, L.A., Dolan, L.M., \& Huang, B. (2012). Timing of stage II lactogenesis is predicted by antenatal metabolic health in a cohort of primiparas. Breastfeeding Medicine, $7(1)$, 43-49. doi: doi: $10.1089 / \mathrm{bfm} .2011,0007$

Panwara, Paritakul, Kasem, Ruangrongmorakot, Wipada, Laosooksathit, Maysita, Suksamarnwong, \& Pawin, Puapornpong. (2016). The effect of ginger on breast milk volume in the early postpartum period: A randomized, double-blind controlled trial. Breastfeeding Medicine, 11(7), 361-365. doi: $10.1089 / \mathrm{bfm} .2016 .0073$.

Patel, S. R., Weng, J., Rueschman, M., Dudley, K. A., Loredo, J. S., Mossavar-Rahmani, Y., .. . Wang, R. (2015). Reproducibility of a standardized actigraphy scoring algorithm for sleep in a US Hispanic/Latino population. Sleep, 38(9), 1497-1503. doi: 10.5665/sleep. 4998

Perez-Escamilla, R., \& Chapman, D. J. (2001). Validity and public health implications of maternal perception of the onset of lactation: an international analytical overview. The Journal of Nutrition, 13I(11), 3021S-3024S.

Preusting, I., Brumley, J., Odibo, B., Spatz, D. L., \& Louis, J. M. (2017). Obesity as a predictor of delayed lactogenesis II. Journal of Human Lactation, 33(4), 684-691.

Qiu, C., Enquobahrie, D., Frederick, I., Abetew, D., \& Williams, M. (2010). Glucose intolerance and gestational diabetes risk in relation to sleep duration and snoring during pregnancy: $A$ pilot study. BMC Womens Health, $10(1), 17$.

Ramiandrasoa, C., Castinetti, F., Raingeard, I., Fenichel, P., Chabre, O., Brue, T., \& Courbiere, B. (2013). Delayed diagnosis of Sheehan's syndrome in a developed country: A retrospective cohort study. European Journal of Endocrinology, 169(4), 431-438. doi: 10.1530/eje-13-0279

Rasmussen, K.M., \& Kjolhede, C.L. (2004). Prepregnant overweight and obesity diminish the prolactin response to suckling in the first week postpartum. Pediatrics, $113(5)$, e465-471. doi: 10.1542/peds.113.5.e465

Rawal, S., Hinkle, S. N., Zhu, Y, Albert, P. S., \& Zhang, C. (2017). A longitudinal study of sleep duration in pregnancy and subsequent risk of gestational diabetes: Findings from a prospective, multiracial cohort. American Journal of Obstetrics \& Gynecology, 216(4), 399 e391-399 e398. doi: 10.1016/j. ajog.2016.11.1051

Reid, K. J., Facco, F. L., Grobman, W. A., Parker, C. B., Herbas, M., Hunter, S., . . Zee, P. C. (2017). Sleep during pregnancy: The nuMoM2b pregnancy and sleep duration and continuity study. Sleep, 40(5). doi: 10.1093/sleep/zsx045

Reutrakul, S., Zaidi, N., Wroblewski, K., Kay, H. H., Ismail, M., Ehrmann, D. A., \& Van Cauter, E. (2011). Sleep disturbances and their relationship to glucose tolerance in pregnancy. Diabetes Care, 34(11), 2454-2457. doi: 10.2337/dc11-0780

Schoenborn, C.A., Adams, P.F., \& Peregoy, J.A. (2013). Health behaviors of adults. United States, 2008-2010. National Center for Health Statistics. Vital Health Statistics, 10(257).

Straus, L. D., Drummond, S. P., Nappi, C. M., Jenkins, M. M., \& Norman, S. B. (2015). Sleep variability in military-related PTSD: A comparison to primary insomnia and healthy controls. Joumal Traumatic Stress, 28(1), 8-16. doi: 10.1002/ jts. 21982

Tsai, S., Lee, P., Lin, J., \& Lee, C. (2016). Cross-sectional and longitudinal associations between sleep and health-related quality of life in pregnant women: A prospective observational study. International Journal of Nursing Studies, 56, 45-53. doi: http:// dx.doi.org/10.1016/j.ijnurstu.2016.01,001

Twedt, R., Bradley, M., Deiseroth, D., Althouse, A., \& Facco, F. (2015). Sleep duration and blood glucose control in women with gestational diabetes mellitus. Obstetrics and Gynecology, 126(2), 326-331. doi: 10.1097/aog.0000000000000959.

Vrieze, S.I. (2012). Model selection and psychological theory: A discussion of the differences between the Akaike Information Criterion (AIC) and the Bayesian Information Criterion (BIC). Psychological Methods, 17(2):228-243. doi: 10.1037/ a0027127.

Wang, H., Leng, J., Li, W., Wang, L., Zhang, C., Li, W., Liu, H., Zhang, S., Chan, J., Hu, G., Yu, Z., \& Yang, X. (2017). Sleep duration and quality, and risk of gestational diabetes mellitus in pregnant Chinese women. Diabetic Medicine, 34(1), 44-50. doi: $10.1111 / \mathrm{dme} .13155$

Wilson, D.L., Fung, A., Walker, S.P., \& Barnes, M. (2013). Subjective reports versus objective measurement of sleep latency and sleep duration in pregnancy. Behavioral sleep medicine 11, 207-221. 